\title{
Article \\ Two-Step $k$-means Clustering Based Information Entropy for Detecting Environmental Barriers Using Wearable Sensor
}

\author{
Bogyeong Lee and Hyunsoo Kim * D
}

Citation: Lee, B.; Kim, H. Two-Step $k$-means Clustering Based Information Entropy for Detecting Environmental Barriers Using Wearable Sensor. Int. J. Environ. Res. Public Health 2022, 19, 704. https:// doi.org/10.3390/ijerph19020704

Academic Editors: David Chapman and Agneta Larsson

Received: 12 November 2021

Accepted: 5 January 2022

Published: 8 January 2022

Publisher's Note: MDPI stays neutral with regard to jurisdictional claims in published maps and institutional affiliations.

Copyright: (C) 2022 by the authors. Licensee MDPI, Basel, Switzerland. This article is an open access article distributed under the terms and conditions of the Creative Commons Attribution (CC BY) license (https:// creativecommons.org/licenses/by/ $4.0 /)$.
Department of Architectural Engineering, Dankook University, 152 Jukjeon-ro, Suji-gu, Yongin-si 16890, Korea; bglee_@dankook.ac.kr

* Correspondence: hkim13@dankook.ac.kr

\begin{abstract}
Walking is the most basic means of transportation. Therefore, continuous management of the walking environment is very important. In particular, the identification of environmental barriers that can impede walkability is the first step in improving the pedestrian experience. Current practices for identifying environmental barriers (e.g., expert investigation and survey) are time-consuming and require additional human resources. Hence, we have developed a method to identify environmental barriers based on information entropy considering that every individual behaves differently in the presence of external stimuli. The behavioral data of the gait process were recorded for 64 participants using a wearable sensor. Additionally, the data were classified into seven gait types using two-step $k$-means clustering. It was observed that the classified gaits create a probability distribution for each location to calculate information entropy. The values of calculated information entropy showed a high correlation in the presence or absence of environmental barriers. The results obtained facilitated the continuous monitoring of environmental barriers generated in a walking environment.
\end{abstract}

Keywords: walkability; environmental barrier; $k$-means clustering; inertial measurement unit (imu); information entropy; wearable sensor

\section{Introduction}

Physical activity is important for both physical and mental well-being [1]. Among various physical activities, walking is the most basic activity [2,3]. It is also the most basic means of transportation. Additionally, walking allows people to stay healthy in their daily lives. An individual uses his body while walking resulting in direct interaction with the external environment [4]. In other words, a person's walking is influenced by the external environment or built environment [5].

Considering that human behavior is affected by the conditions of the external environment while walking [6], the need to continuously manage the urban or built environment is increasing [7]. In particular, it is essential to provide a pedestrian-friendly walking environment. The term "pedestrian-friendly walking environment" may indicate an environment with high walkability [8]. The term "walkability" can be defined in various ways since the term includes many key themes or dimensions such as traversable environments, compact places, being safe for walking, physically-enticing environments, lively and sociable environments, an environment providing sustainable transportation options, and exercising-inducing environment [9]. Thus, walkability is determined by the combination of these dimensions. In addition, in the urban design domain, walkability cannot be measured by a just single dimension [10]. Although the concept "walkability" is very complicated because overall walkability of a specific street is the combination result of multi-dimensional features, it is clear that environmental barriers-an environmental feature makes pedestrians feel uncomfortable during walking — should be identified and eliminated to improve walkability [11,12]. There are diverse definitions of environmental barriers [13-15]. Even more, environmental barriers vary with age, gender, and sociocultural contexts $[15,16]$. Environmental barriers in walking can include fixed (e.g., broken 
pavement, insufficient width, etc.) and unfixed features (e.g., vehicles, traffic volume, complexity, etc.) [10]. Considering that walking and gait are a result of an interaction between surfaces and human body, environmental barriers in this study are an environmental feature that can make pedestrians uncomfortable in a specific location (e.g., sidewalks and crosswalks). As shown in the previous study [17], this study uses a very narrow concept of walkability defined as how much regular gait patterns are maintained.

In existing research, many approaches (i.e., surveys by experts, surveys by actual users, reporting of complaints by civilians, walking audits, and walking interviews, etc.) have been developed to identify environmental barriers [18-21]. Although these methods have significantly improved the walking environment by identifying various environmental barriers, the following problems exist. Based on the results of surveys by experts, users of the actual walking environment are usually excluded from the evaluation stage. In addition, there is a possibility of subjective bias in the evaluation by experts [12], and continuous evaluation requires excessive financial expenditure [11]. However, in surveys by actual users, users of the walking environment can directly participate in the evaluation; however, a bias may exist depending on the sampling of people participating in the survey [22]. In addition, continuous monitoring is difficult because the evaluation of walking environment is performed at intervals [23]. Lastly, in the case of handling civilian complaints, the opinions of the complainants cannot be regarded as the opinions of the majority of pedestrians. Additionally, the opinions of civil petitioners can be prioritized in the budget execution process $[24,25]$. Summarizing the aforementioned problems: (1) Users of the walking environment are excluded from the evaluation process, (2) there is a possibility of subjective bias in the evaluation process, and (3) continuous evaluation is difficult due to problems such as budget and time.

Recent developments in sensing technology can provide an opportunity to solve the above-mentioned problems. People-centric sensing involves collecting and analyzing data with citizens as sensors [26]. In other words, it is possible to monitor various conditions (such as crowd detection, event detection, noise, traffic, etc.) of the built environment using data acquired from the daily lives of citizens [26-30]. People-centric sensing has also been used to identify environmental barriers. Existing studies have attempted to identify factors that cause discomfort by analyzing data generated from people's daily lives [31-33].

The premise of existing studies is that there is a change in human behavior or response in the presence of external stimuli. Kim et al. [12] conducted a study to identify defects on sidewalks using pedestrian data collected from an inertial measurement unit (IMU). Zeile [34] developed a method to measure walkability by combining the data from surveys, biosensors, and geospatial analysis. Ahn et al. [35] conducted a study to identify environmental distress factors using electrodermal activity (EDA) and IMU. Kim et al. [36] proposed a method to evaluate the neighborhood built environment using EDA, gait patterns, and blood volume pulse. In addition, Lee et al. [23] conducted a study to determine environmental barriers based on the stress measured by a wearable sensor. Zanwar et al. [37] used wearable sensors to identify barriers that were not suitable for the elderly and disabled. Existing studies describe various methods to identify the factors that can inhibit walkability. However, these studies dealt only with the relationship between the point where the intensity of the pedestrian's reaction is high and the existence of environmental barriers. In existing studies, the intensity of response means that the value of acceleration through the IMU is high [12], that the measured value of EDA appears high [35], and that the blood volume pulse is measured as high [36]. However, even at a point where the intensity of the reaction is low, an environmental barrier may exist. As shown in a previous study [38], when the floor is slippery in a walking environment, the value of acceleration through IMU can be measured as low by reducing the stride length or reducing the walking speed in response to this. Therefore, it is necessary to include these various reactions in the identification process of environmental barriers.

Human behavior is very complex, and the response to external stimuli (especially environmental barriers that can impair walkability) may differ for each individual. According 
to a study by Kim et al. [38], ironworkers showed various reactions while responding to hazards. The experimental results indicated that the strength of the reaction was negligible for a strong external stimulus. A notable point was that there were diverse gait patterns (low normality) regarding a construction hazard. On a slippery surface, some workers tried to slow down their gait speed, and others slipped. In another study for detecting defective sidewalk (finding cracks, holes, and vertical separations) [12], pedestrians also showed diverse responses to defects (low normality). On the other hand, pedestrians' gait patterns were highly maintained under normal conditions (without sidewalk defects). In a study by Kim [39], when a car passes around a pedestrian on a road with a mixture of sidewalks and lanes, pedestrians show very low intensity of responses or stop and wait for the vehicle to pass. Conversely, there were pedestrians who took immediate action to avoid contact with vehicles. When environmental barriers act, the unpredictable characteristics of the collective responses of pedestrians is highly dispersed. According to information entropy theory, high unpredictability contains more information and has high entropy.

In many previous studies, entropy theory has been used to measure walkability [40]. Related studies calculated walkability by calculating entropy according to the degree of land use mix [41-43]. In addition, research in fields, such as crowd safety [44], emergence [45], pedestrian flow [46], evasive actions of pedestrians [47], and abnormal pedestrian detection [48] through the entropy of pedestrian behavior have also been conducted.

Considering the advantages of using wearable sensor and information entropy in existing studies and the irregular changes in the behavior of pedestrians at a specific location, the existence of an environmental barrier can also be estimated through the degree of entropy of the observed behavior. This study is conducted to achieve the following objectives: (1) developing an information entropy calculation method that can quantify pedestrians' diverse responses; (2) testing the suggested method in an actual walking environment; (3) comparing the calculated information entropy values and environmental barriers; and (4) confirming that the method proposed in this study is feasible.

\section{Methodology}

\subsection{Experimental Design}

To achieve the purpose of this study, a certain number of participants were recruited and wearable sensors were attached to them. They were instructed to walk in the experimental site. Figure 1 shows the experimental site used in this study. The total length of the test site is $1.80 \mathrm{~km}$. It is divided into 12 sections according to the characteristics and continuity. The best representation of each section is shown in Figure $1 \mathrm{~b}-\mathrm{m}$, and the order of each figure follows the section number.
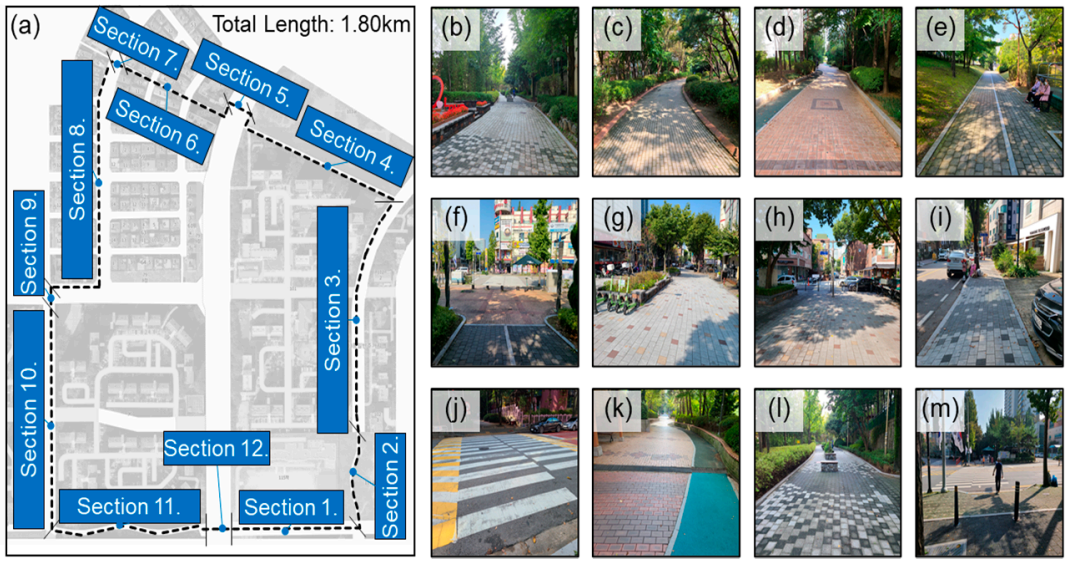

Figure 1. Overview of experimental site; (a) Site and sections and (b-m) representative pictures of each section. 
The experimental site is a district in Bundang-gu, Seongnam-si, Gyeonggi-do, Korea. The reason for selecting this area is its diversity of walking environments. We have closely examined the actual pedestrian interaction in various environments and identified the environmental factors that cause various behavioral responses. The study area includes parks, well-maintained roads, crosswalks, residential areas, and commercial areas. In addition, it was expected that a sufficient number of participants could be recruited from the area to secure sufficient statistical significance because the experimental area is a residential area. Table 1 shows the description, length, and average width of each section.

Table 1. Summary of characteristics of each section.

\begin{tabular}{|c|c|c|c|}
\hline Section Number & Description & Length (m) & Avg. Width (m) \\
\hline 1 & $\begin{array}{l}\text { Well-maintained sidewalk blocks } \\
\text { Slight uphill road }\end{array}$ & 171 & 4.8 \\
\hline 2 & $\begin{array}{l}\text { Well-maintained sidewalk blocks } \\
\text { installed for over } 10 \text { years }\end{array}$ & 118 & 2.4 \\
\hline 3 & $\begin{array}{l}\text { Well-maintained sidewalk blocks } \\
\text { Slight downhill }\end{array}$ & 275 & 4.5 \\
\hline 4 & $\begin{array}{l}\text { Well-maintained sidewalk blocks in } \\
\text { a park installed for over } 10 \text { years }\end{array}$ & 219 & 2.6 \\
\hline 5 & $\begin{array}{l}\text { Crossing on a six-lane road (with } \\
\text { traffic lights) }\end{array}$ & 26 & 4.8 \\
\hline 6 & $\begin{array}{l}\text { Well-maintained sidewalk blocks } \\
\text { Crossings on two-lane roads (no } \\
\text { traffic lights) }\end{array}$ & 114 & 6.2 \\
\hline 7 & $\begin{array}{l}\text { Crossings on four-lane roads (no } \\
\text { traffic lights) }\end{array}$ & 11 & 8 \\
\hline 8 & $\begin{array}{l}\text { Mixed residential and commercial } \\
\text { spaces } \\
\text { Illegal parking and piled materials } \\
\text { on sidewalks }\end{array}$ & 324 & 1.8 \\
\hline 9 & $\begin{array}{l}\text { Crossings on four-lane roads (with } \\
\text { traffic lights) }\end{array}$ & 13 & 8 \\
\hline 10 & $\begin{array}{l}\text { Well-maintained sidewalks and } \\
\text { surrounding facilities }\end{array}$ & 274 & 4.8 \\
\hline 11 & $\begin{array}{l}\text { Well-maintained sidewalks and } \\
\text { surrounding facilities }\end{array}$ & 231 & 4.8 \\
\hline 12 & $\begin{array}{l}\text { Crossing on a six-lane road (with } \\
\text { traffic lights) }\end{array}$ & 26 & 4.8 \\
\hline
\end{tabular}

A total of 64 participants were recruited to conduct the experiments. Participants of various age groups who were healthy enough to participate in the experiment were recruited from the sports clubs (running club and senior gateball club) around the experimental site. The age and gender of the participants are shown in Table 2. None of them had any discomfort while walking or any physical constraint in performing the walking experiment for a distance of $1.8 \mathrm{~km}$.

Table 2. Age and gender of participants.

\begin{tabular}{cccc}
\hline Age & Male & Female & Total \\
\hline $20 \mathrm{~s}-30 \mathrm{~s}$ & 16 & 13 & 29 \\
$40 \mathrm{~s}-50 \mathrm{~s}$ & 8 & 8 & 16 \\
Over 60 s & 10 & 7 & 17 \\
Total & 34 & 28 & 64 \\
\hline
\end{tabular}


Prior to the experiment, all participants were informed that the trial had been approved by the Institutional Review Board (IRB: DKU 2020-09-027) and that all data would be anonymized and used for research purposes only. The experiment was performed in two stages. In the first stage, the participants were instructed to wear an IMU sensor on their right ankle and walk at a comfortable velocity along a set route (starting at Section 1 and finishing at Section 12). In the second stage, the participant and experimenter walked along the path together to examine and record the points at which the participant experienced an environmental barrier during the first stage of the experiment. In addition, a sufficient break (10-20 min) was provided between the experiments to recover from fatigue. The recorded information was used to analyze the relationship between the existence of an environmental barrier and information entropy values. Because a trial (conducting a participant's experiment) takes about one hour, the experiment was conducted for 16 groups of 4 individuals over 27 days from 4 October to 30 October 2021 (excluding weekends and rainy days). During the experiment, the temperature was $14-20{ }^{\circ} \mathrm{C}$ and humidity was $25-40 \%$.

\subsection{Research Framework}

Figure 2 depicts the research framework proposed in this study. It consists of three major steps. First is data collection. Three devices are used to collect the data. First, the camcorder is used to confirm the ground truth and is attached to the participant's chest. Second, a smartphone is placed in the front pocket of the participant's trousers. Smartphones are used to collect GPS data during the experiment. Third, an IMU sensor is attached to the participant's right ankle. During the experiment, the IMU sensor measures 3-axis acceleration and 3-axis angular velocity. In this study, data collected from camcorder and smartphone are not used to calculate information entropy but only for ground truth and GPS location.

The second step is to classify various gaits using $k$-means clustering. Before extracting individual gait features, gait detection should be performed on the 3-axis acceleration and 3-axis angular velocity data obtained through the IMU sensor. In this study, gait detection was performed using an algorithm proposed by $\mathrm{O}^{\prime}$ Connor et al. [49]. Based on the six types of data collected from the IMU, the gait of each participant is detected based on heel strike and toe off. $k$-means clustering is used to distinguish between normal and abnormal gaits in the first clustering, and abnormal gaits are classified into six types in the second clustering.

Finally, the seven types of gait (one normal gait and six types of abnormal gait) are classified according to the measured location and a behavioral response distribution is created for each location. Based on this distribution, the information entropy for each section is constructed. The calculated information entropy values are verified against the location of the environmental barrier.

\subsection{Calculation Process of Information Entropy by Location}

As mentioned earlier, $k$-means clustering was performed twice in this study because two groups were classified (normal and abnormal gait) after the first clustering. To construct a detailed behavioral response distribution, we have re-clustered the groups classified as abnormal gaits in the first $k$-means clustering.

Since each detected gait includes various values, we have replaced six types of data with one value according to the gait. The average of the signal vector magnitude (SVM) is calculated to obtain the average of the intensities of six types of data appearing in one gait as represented in Equation (1):

$$
S V M_{i j}=\left[\sum_{k=1}^{n} \sqrt{x_{k}^{2}+y_{k}^{2}+z_{k}^{2}}\right] / n
$$


where $n$ is the total number of IMU measurements of the $j$ th participant on the $i$ th grid cell, $x_{k}$ is the $k_{\mathrm{th}}$ acceleration (or $k$ th angular velocity) of the anterior-posterior axis, $y_{k}$ is the $k$ th acceleration (or $k$ th angular velocity) of the horizontal axis, and $z_{k}$ is the $k$ th acceleration (or $k$ th angular velocity) of the vertical axis.

The SVM values for each gait obtained using Equation (1) were normalized indicating that one gait has two normalized SVM values and clustering is performed based on these values. First $k$-means clustering is performed to classify gaits as normal and abnormal. Second $k$-means clustering is performed to classify only abnormal gaits. Finally, a behavioral response distribution is created based on seven types of gait.

The established behavioral response distribution by location is used to calculate information entropy. According to information theory, the entropy of a random variable is the average level of information [50]. This information is also the level of occurrence of an unexpected event [51]. Shannon [52] introduced the concept of information entropy to quantify the uncertainty of a random variable. In this study, the Shannon entropy (SE) can be represented by a combination of the probabilities of seven types of activity classified by two-step $k$-means clustering. SE can be calculated using Equation (2) as:

$$
H(A)=-\sum_{i=1}^{n} p\left(a_{i}\right) \log p\left(a_{i}\right)
$$

where $H(A)$ is the entropy and $p\left(a_{i}\right)$ is the probability distribution.
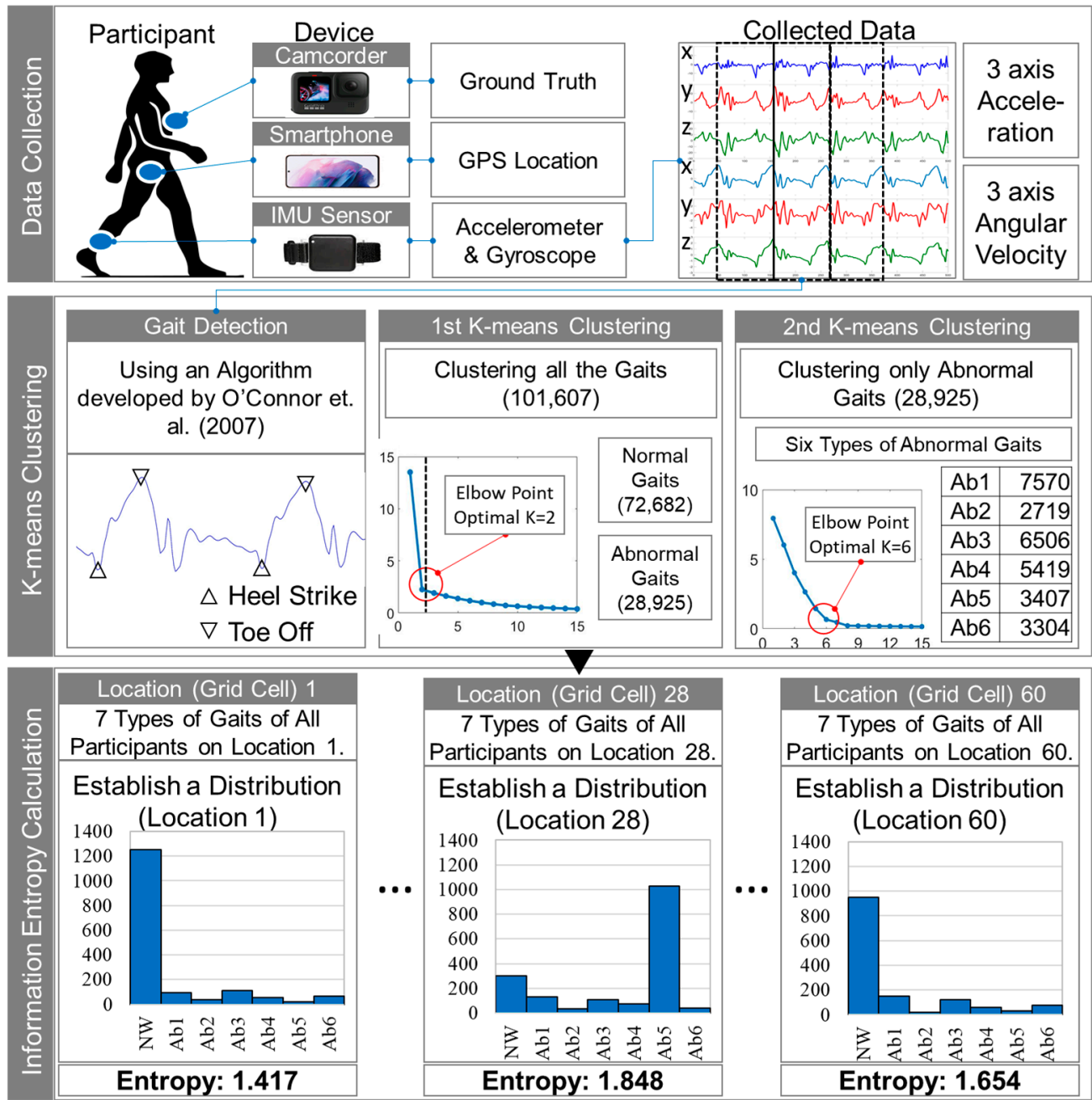

Figure 2. Research framework. 


\section{Results}

\subsection{Environmental Barriers Identified by Participants' Expression: Ground Truth}

Figure 3 shows the specific points of environmental barrier as expressed by the participants in this study. After the first walking experiment, the participant and experimenter walked along the experimental route again and recorded the points indicated by the participant as an environmental barrier. To identify the environmental barriers indicated by 64 participants a point recorded as an environmental barrier more than six times (about $10 \%$ of the participants) was defined as an environmental barrier. Based on this, a total of nine environmental barriers were selected in this study.
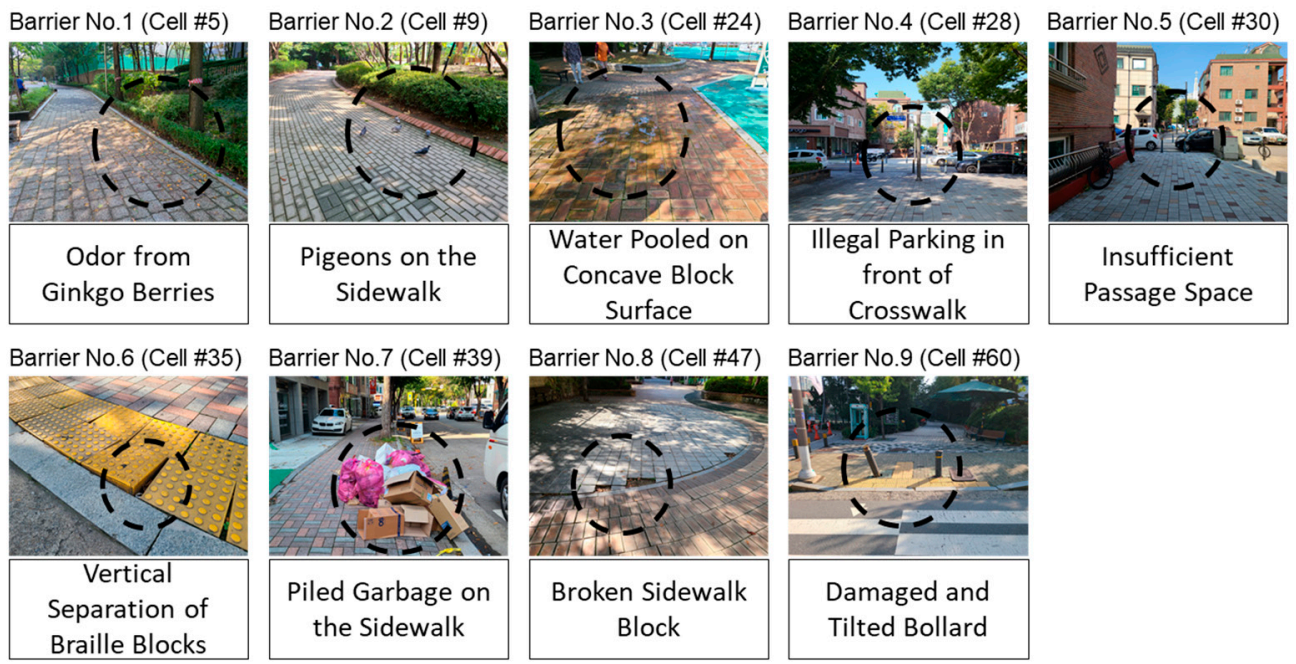

Figure 3. Captured environmental barriers as indicated by participants.

The identified environmental barriers can be classified according to their occurrence characteristics and continuity as follows: (1) seasonal environmental barrier (barrier number 1), (2) temporal environmental barrier (barrier numbers 2 and 3), (3) human-made environmental barrier (barrier numbers 4, 5, and 7), and (4) physically disordered environmental barrier (barrier numbers 6, 8, and 9). Although the seasonal environmental barriers showed no significant difference from the surrounding environment, the participants tended to avoid it because of the odor of Ginkgo biloba. The temporal environmental barrier was not expressed as an environmental barrier by the participants if there was no pigeon or the water was not stagnant while performing the experiment. In other words, an element can act as an environmental barrier for a relatively short time. Human-made environmental barriers were due to illegal parking. However, the participants did not express discomfort when there was no illegal parking in the area. Lastly, the physical disordered environmental barrier was considered as an environmental barrier only by those who passed through the physical disorder. In the following sections, the process and feasibility of the identification of the above-mentioned environmental barriers using information entropy are explained.

\subsection{Two-Step k-means Clustering Results}

In this study, the total number of gaits collected from 64 participants was 101,607. First, $k$-means clustering was performed for the entire gait. Next, the sum of the squared distances according to the value of $K$ was calculated to obtain the optimal number of clusters. The optimal value of $K$ was determined to be 2 based on the elbow point (see Figure $4 \mathrm{a}$ ). When a $K$ value of 2 was applied, the 72,682 gaits were classified into with normal gait, and 28,925 gaits were classified into abnormal gait. 


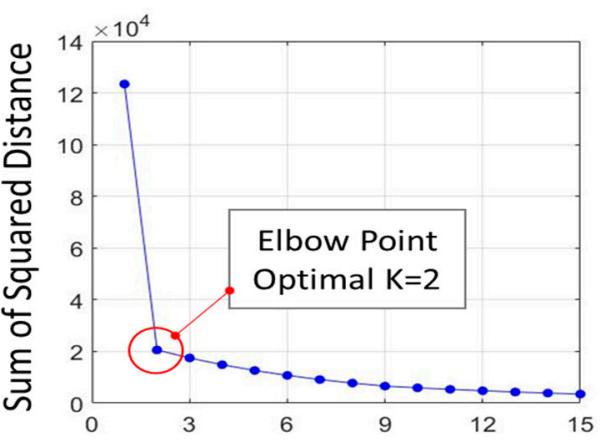

(a)

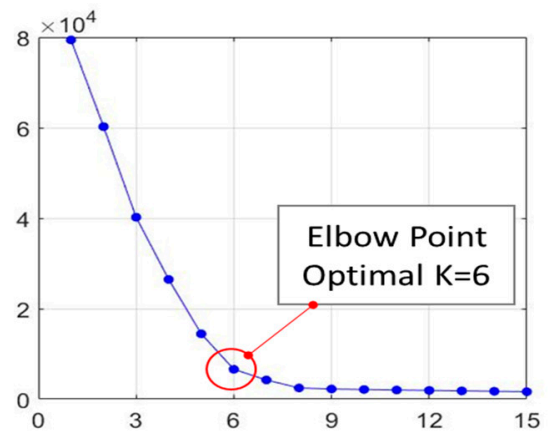

(b)

Figure 4. Optimal values of $k$-means clustering: (a) First clustering for all gaits and (b) second clustering for only abnormal gaits (excluding normal gaits).

Considering that people's reactions to various external environments are also diverse, it is necessary to consider the reactions of pedestrians due to environmental barriers. Therefore, $k$-means clustering was performed again on only 28,925 abnormal gaits classified previously. The optimal $K$ value in the second cluster was found to be 6 .

Table 3 shows the results of classification of 101,607 gaits into 7 groups along with the levels of acceleration and angular velocity. In the normal gait group, both acceleration and angular velocity are at average levels. In the abnormal gait 1 group, both values are classified as "Low" level. In the abnormal gait 2 group, acceleration is "Low" level and angular velocity is "High" level whereas the abnormal gait 5 group indicated the opposite. In addition, in the abnormal gait 3 and 4 groups, both acceleration and angular velocity are high (very high or high). Finally, in the abnormal gait 6 group, both values are very low. The number of gaits included in each group is calculated as the probability of the corresponding event. Based on the probabilities of the seven types of gait, a behavioral response distribution was created for each cell.

Table 3. Characteristics of classified normal gaits and six types of abnormal gait in terms of accelerometer and gyroscope data.

\begin{tabular}{cccc}
\hline Gait Type & Number of Gaits & $\begin{array}{c}\text { Mean of Normalized } \\
\text { SVM of Acceleration }\end{array}$ & $\begin{array}{c}\text { Mean of Normalized } \\
\text { SVM of Angular Velocity }\end{array}$ \\
\hline Normal Gait & 72,682 & Moderate & Moderate \\
Abnormal Gait 1 & 7570 & Low & Low \\
Abnormal Gait 2 & 2719 & Low & High \\
Abnormal Gait 3 & 6506 & High & Very High \\
Abnormal Gait 4 & 5419 & Very High & High \\
Abnormal Gait 5 & 3407 & High & Low \\
Abnormal Gait 6 & 3304 & Very Low & Very Low \\
\hline
\end{tabular}

\subsection{Comparison of Distribution by Cells with/without an Environmental Barrier}

Figure 5 shows the behavioral response distribution for the 12 cells that represent the environmental barrier indicated by the participants and three cells under normal conditions. Figure 5a-c show the distributions corresponding to cell numbers 1, 21, and 41, respectively. In cells that do not contain an environmental barrier, the ratio classified as normal walking by $k$-means clustering is $76.60 \%$ (66,141 gaits), and the ratio of behaviors corresponding to $\mathrm{AB} 1-\mathrm{AB} 6$ is $23.40 \%$ (20,208 gaits). It was observed that, in cells that do not contain an environmental barrier, the participants walked normally at a very high rate. For cells that contain the environmental barrier as shown in Figure $5 d-1$, the ratio of normal walking is $42.87 \%$ (6541 gaits), and the ratio of behaviors corresponding to $\mathrm{AB} 1-\mathrm{AB} 6$ is $57.13 \%$ (8717 gaits). 

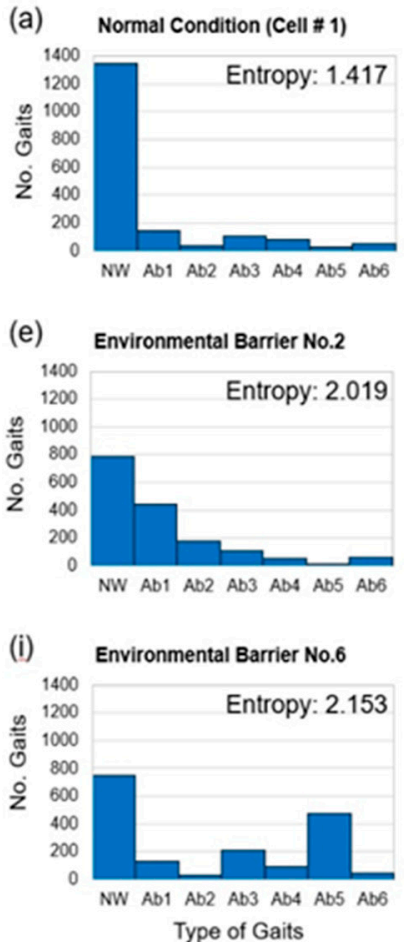

(b)

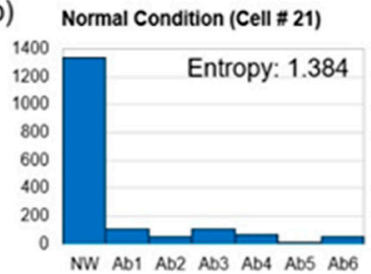

(f)

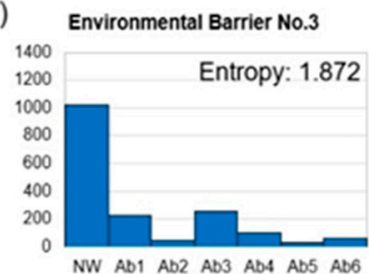

(j)

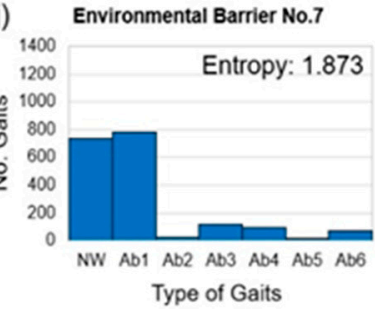

(c)

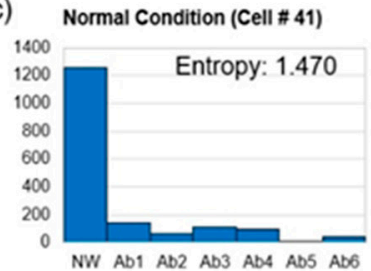

(g)

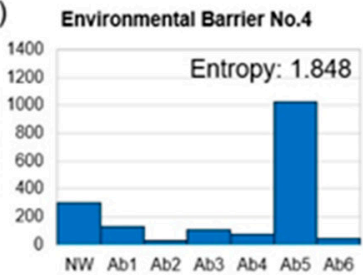

(k)

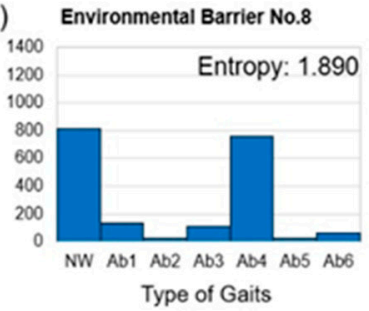

(d)

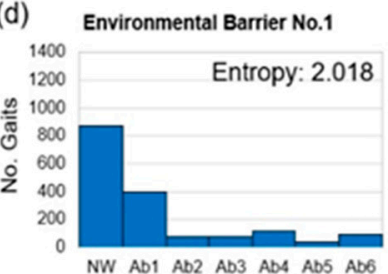

(h)

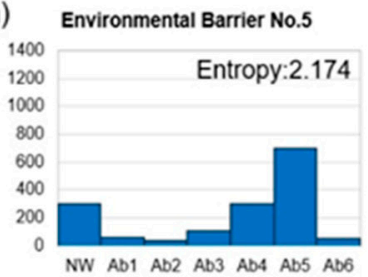

(l)

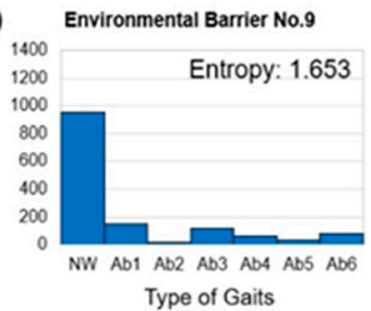

Figure 5. Behavioral response distribution: $(\mathbf{a}-\mathbf{c})$ Behavioral response distribution during normal conditions (Cell Number 1, 21, and 41, respectively) and (d-1) behavioral response distribution (Cell Number 5, 9, 24, 28, 30, 35, 39, 47, and 60, respectively).

As Equation (2), it can be seen that the higher the occurrence rate of the rare event, the higher the value of entropy. In order to respond to environmental barriers, pedestrians take the most appropriate or familiar responses to themselves. Due to individual differences, the types of responses that pedestrians can choose can also be diversified, so a high information entropy value is observed at the environmental barrier. On the other hand, in the normal condition, the ratio of normal gait is observed to be very high because the pedestrian has no difficulty in maintaining his/her gait. Therefore, it can be seen that the information entropy is low in normal condition.

On comparing the shape of distribution, it can be observed that the form of behavioral response distribution appearing in each environmental barrier is different. However, behavioral distribution of environmental barrier numbers 1 and 2 is similar. To statistically analyze the shape of each cell, the behavior of cells that do not contain an environmental barrier (NC in Table 4) and nine cells that contain an environmental barrier (EB1-9 in Table 4) are analyzed. Since there are 51 cells that do not contain an environmental barrier, the average information entropy values of seven types of gait corresponding to cells that do not contain an environmental barrier was used to perform a $t$-test for pairwise comparison. Table 4 shows the results of $t$-tests. All $t$-test results except for environmental barrier numbers 1 and $2(p$-value $=0.079)$ show statistical significance $(\alpha=0.05)$.

\subsection{Information Entropy Values by Location}

Information entropy was calculated based on the behavioral response distribution of each cell as presented in the previous section. Figure 6 shows the information entropy values and environmental barriers according to the location. This is consistent with the peak values of the nine environmental barriers and information entropy values suggested by the participants in this experiment. This indicates that environmental barriers result in various reactions from pedestrians. 
Table 4. $t$-test results of pairwise comparison among cells with/without an environmental barrier.

\begin{tabular}{ccccccccccc}
\hline$p$-Value & NC & EB1 & EB2 & EB3 & EB4 & EB5 & EB6 & EB7 & EB8 & EB9 \\
\hline NC & - & - & - & - & - & - & - & - & - \\
EB1 & $<0.001$ & - & - & - & - & - & - & - & - \\
EB2 & $<0.001$ & 0.079 & - & - & - & - & - & - & - \\
EB3 & $<0.001$ & $<0.001$ & $<0.001$ & - & - & - & - & - & - \\
EB4 & $<0.001$ & $<0.001$ & $<0.001$ & $<0.001$ & - & - & - & - & - \\
EB5 & $<0.001$ & $<0.001$ & $<0.001$ & $<0.001$ & $<0.001$ & - & - & - & - \\
EB6 & $<0.001$ & $<0.001$ & $<0.001$ & $<0.001$ & $<0.001$ & $<0.001$ & - & - & - \\
EB7 & $<0.001$ & $<0.001$ & $<0.001$ & $<0.001$ & $<0.001$ & $<0.001$ & $<0.001$ & - & - \\
EB8 & $<0.001$ & $<0.001$ & $<0.001$ & $<0.001$ & $<0.001$ & $<0.001$ & $<0.001$ & $<0.001$ & - \\
EB9 & $<0.001$ & $<0.001$ & $<0.001$ & $<0.001$ & $<0.001$ & $<0.001$ & $<0.001$ & $<0.001$ & $<0.001$ & - \\
\hline
\end{tabular}

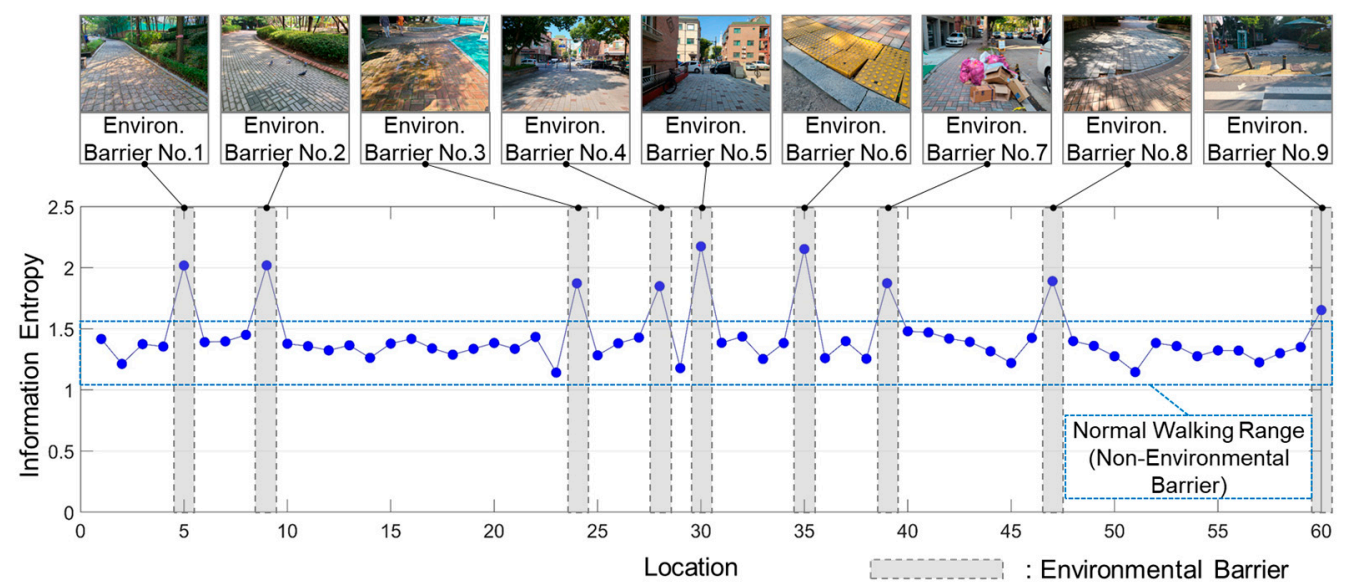

Figure 6. Information entropy value by location.

To confirm the feasibility of the proposed method, the relationship between calculated information entropy values and existence of environmental barriers was measured. The information entropy values of the cell are continuous variables whereas the existence of environmental barriers can be represented as a binary variable (existence as 1 and absence as 0). To investigate the relationship between the existence of an environmental barrier and information entropy in a statistical manner, we have used the point biserial correlation coefficient. This is generally used when one variable is continuous and the other is dichotomous $[53,54]$. Additionally, the values that relate to the existence of the environmental and information entropy values were calculated using Equation (3) as follows:

$$
r_{p b}=\frac{\left(M_{1}-M_{0}\right) \sqrt{\left.n_{1} n_{0} / n^{2}\right)}}{\sqrt{\frac{1}{n}} \sum_{i=1}^{n}\left(X_{1}-\bar{X}\right)^{2}}
$$

where $r_{p b}$ is the point biserial correlation coefficient, $M_{1}$ is the mean value of the continuous variable $X$ (information entropy values) for all data points in group 1 (existence of environmental barrier), $M_{0}$ is the mean value of the continuous variable $X$ (information entropy values) for all data points in group 2 (absence of environmental barrier), $n_{1}$ is the number of data points in group $1, n_{0}$ is the number of data points in group 2 , and $n$ is the total sample size.

Based on the point biserial correlation, the correlation between the existence of an environmental barrier and information entropy values was calculated as $0.759(\alpha<0.05$, $p=0.001$ ) According to previous studies [49,50], a correlation coefficient greater than 0.7 indicates a high degree of correlation. The results showed that the information entropy and existence (presence and absence) of environmental barriers are highly correlated.

Additionally, it was observed that the average value of information entropy of 60 cells was 1.432 , maximum value was 2.174 , and minimum value was 1.142 . Based on the presence 
or absence of an environmental barrier, the average value of cells that did not contain an environmental barrier was 1.342 , maximum value was 1.480 , and minimum value was 1.142. However, the average value of cells that consisted of an environmental barrier was 1.945 , maximum value was 2.174, and minimum value was 1.653 .

Based on the results of the experiments conducted in this study, the threshold for measuring the existence of environmental barriers was set to 1.500 . That is, when the information entropy value is less than 1.5 , the environment is favorable for normal walking, and when the information entropy value is 1.5 or more, there is a possibility of the existence of an environmental barrier in the corresponding location.

Although the threshold of information entropy that can identify environmental barriers is set to 1.5, this value can vary depending on the size of individual cells and number of gait types constituting the distribution. In this study, each cell had a dimension of $30 \mathrm{~m}$. The reason for choosing $30 \mathrm{~m}$ as the cell size was to secure a sufficient number of walks to obtain information entropy in one cell. In general, since one gait has a length of about $1.5 \mathrm{~m}$, it was possible to obtain at least 20 walks in one cell. This is because, if there is a sufficient number of gaits, one or two abnormal gait patterns can be prevented from excessively affecting the information entropy calculation process. Unless the size of the environmental barrier was very large, the range occupied by it at a distance of $30 \mathrm{~m}$ was not large. Therefore, even if there is an environmental barrier, many gaits were still classified as normal walking. This is because, the ratio of normal walking varies with the distance of the section, and the value of threshold presented above may change. In addition, seven gait types were used to estimate information entropy. The range of information entropy values is affected by the number of gait types used. Therefore, if the number of gait types is changed, the threshold value also changes.

\section{Discussion}

\subsection{Contributions of the Suggested Method}

The proposed method is used to quantify the behavior of pedestrians in response to environmental barriers using information entropy. Information entropy is calculated using probability distribution. To obtain this distribution, six types of data (3-axis acceleration and 3-axis angular velocity) acquired through the IMU sensor were used to monitor minute changes in the gait process. Six types of data appearing in the gait process were clustered and classified into seven types of gait, which can be also explained as follows: (1) normal gaits are normal walking; (2) abnormal gait 1 is smooth detour; (3) abnormal gait 2 is sharp detour; (4) abnormal gait 3 is jumping over a barrier; (5) abnormal gait 4 is stepping a barrier with caution; (6) abnormal gait 5 is stepping a barrier without caution; and (7) abnormal gait 6 is stop and go. In addition, the reason for using seven types of walking is to include more diverse responses in the information entropy calculation process. Information entropy assumes that rarer events have more information. That is, if only normal gaits occupying the majority and abnormal gaits (just using two clusters) are used, the ability to identify environmental barriers may be reduced. When seven types of gait were used in "3.4 Information entropy values by location" Section, the point biserial correlation coefficient was 0.759 (using seven types of gaits). If six types of abnormal gait are not used and all six abnormal gait types are combined as only one type of abnormal gait, the point biserial correlation coefficient is 0.529 (two types of gait, $\alpha<0.05, p=0.001$ ). Considering these results, using more diverse gait types can be advantageous in identifying environmental barriers. Existing studies have conducted research to identify environmental barriers or hazards using only two normal and abnormal gaits [55,56]. Although barriers or hazards can be identified only with the existing binary classification-based approach, the method proposed in this study shows that more detailed characteristics can be inferred by classifying behaviors into subtypes regarding environmental barriers.

In addition, the two-step $k$-means clustering approach proposed in this study shows that six types of abnormal gait can be distinguished. When clustering only abnormal gaits, the classification performance is higher than when all normal gaits are included. This is 
because the ratio corresponding to normal gaits is high when calculating the centroid in the process of performing $k$-means clustering for all gaits; hence, an abnormal gait group with a relatively small number of gaits (classified as abnormal gait groups 1-6) of centroids may not be clearly visible. Abnormal gaits can be classified according to their characteristics using two-step $k$-means clustering. As a result, these behavioral characteristics can be reflected in information entropy. Moreover, the gait types classified in this study can be used to construct machine learning or artificial intelligence-based systems in the future. When new data are generated, they can be added to the distribution by measuring the similarity with the already classified data, instead of clustering.

Finally, information entropy based on diverse behaviors is highly correlated with the existence of environmental barriers compared to existing intensity-based approaches and two types of behavior-based approach. There are several reasons why information entropy can be advantageous for identifying environmental barriers. First, individual differences including physical and cognitive characteristics may be an important trigger for causing diverse responses to an environmental barrier. Information entropy can represent this diversity well. Various responses to the external environment mean that the amount of information increases; even if only some of the pedestrians show abnormal responses, they are included in the information entropy calculation process and strongly affect this. Compared with normality or average, a small number of abnormal behaviors can sufficiently influence the information entropy calculation process.

\subsection{Future Applications and Benefits of the Suggested Method}

Although the conventional methods (e.g., surveys and evaluation by experts) for identifying environmental barriers have indicated what environmental elements act as a barrier for pedestrians, there are several disadvantages such as sample bias and time and financial costs. To overcome these disadvantages, wearable device-based approaches have been attempted. Nevertheless, existing studies using wearable devices and this study also face problems, in that a sample of respondents (participants) must be recruited, and participants must attach an IMU sensor. To develop a real-world application, there is a need to replace the IMU sensor with a smartphone. Smartphones include various sensors, and the IMU sensor is also built into the device. In addition, smartphones have the advantage of being possessed by most citizens in their daily life.

In order to change the method proposed in this study to fit the real-world situation, it is necessary to make it possible for pedestrians to provide data on their daily life by carrying a smartphone rather than directly attaching the IMU sensor to their body. If data collection through a smartphone is possible, data of pedestrians can be collected in real time. Real-time data collection will enable continuous monitoring of the walking environment. Despite these advantages, there are several hurdles that must be overcome for a people-centric sensing approach to be feasible, including recruiting and sampling participants [57] and protecting the privacy of participants [27]. Although research related to people-centric sensing is still immature, it is expected that the hurdles will be addressed in the near future [58].

\subsection{Limitations and Future Research}

Despite the strength and contribution of the method proposed in this study, there are several limitations. Walkability and environmental barriers are a relative concept [59]. Even more, walkability is the result of a combination of physical features, urban design qualities, and individual reactions $[9,10]$. However, the walkability and environmental barriers in this study do not incorporate all these elements. This study tried to identify features that can make pedestrians' walking patterns dispersed. In consideration of overall walkability, the high normality of gait patterns may not be suitable for developing an attractive street. For example, a pedestrian's gait pattern can be dispersed if there are many attractive elements in a street and a pedestrian pays attention to the elements. Therefore, the results of this study are limited to identifying environmental barriers that are usually 
fixed and inconvenient. Future study needs to investigate a more comprehensive meaning of walkability.

In this study, an experiment was performed by attaching an IMU sensor to the ankle of the participants for gait analysis. In future, the results of this study can be extended to people-centric sensing to promote built environment monitoring of a smart city. Therefore, it is necessary to conduct a behavioral analysis of pedestrians with smartphones rather than attaching a sensor to the ankle. In other words, it is necessary to detect and classify various responses to environmental barriers even while walking with a smartphone in a pocket or in hand. In addition, the Bundang area selected as the experimental site in this study was well managed as a planned city. Hence, it is essential to conduct experiments under various conditions for different types of experimental sites.

\section{Conclusions}

Walking is the most basic physical activity to stay healthy. Therefore, it is essential to create an ideal walking environment by identifying the environmental barriers that can impede walking. Efforts to identify existing environmental barriers are labor-intensive, time-consuming, and discontinuous. Although previous studies have attempted to identify environmental barriers using a wearable sensor and people-centric sensing, they have only focused on the magnitude of responses (e.g., high value of accelerations or EDA) at the barrier and not the reactions of the participants.

In this study, we proposed a method to estimate information entropy by quantitatively measuring various responses to environmental barriers using wearable sensors. To verify the proposed method, we performed an experiment on 64 participants. Based on the data collected from the experiment, seven types of gait were classified according to the responses of the participants through two-step $k$-means clustering. The behavioral response distribution for each location was obtained and information entropy was calculated. The results indicated a high correlation with the presence or absence of environmental barriers. Additionally, it was highlighted that the proposed method is feasible for identifying environmental barriers.

Author Contributions: Conceptualization, B.L. and H.K.; methodology, B.L.; software, B.L.; validation, B.L. and H.K.; investigation, B.L.; resources, B.L.; data curation, B.L.; writing-original draft preparation, B.L. and H.K.; writing-review and editing, H.K.; visualization, B.L.; supervision, H.K. All authors have read and agreed to the published version of the manuscript.

Funding: This study was carried out with the support of 'R\&D Program for Forest Science Technology (Project No. 2021355C10-2123-AC03)' provided by Korea Forest Service (Korea Forestry Promotion Institute). In addition, this research was also supported by the Basic Science Research Program through the National Research Foundation of Korea (NRF), funded by the Ministry of Education (2021R1I1A1A01052305).

Institutional Review Board Statement: The study was conducted according to the guidelines of the Declaration of Helsinki, and approved by the Institutional Review Board of DANKOOK UNIVERSITY (DKU 2020-09-027, date of approval 14 September 2020).

Informed Consent Statement: Informed consent was obtained from all subjects involved in the study.

Data Availability Statement: Some or all data, or code generated during the study are proprietary or confidential in nature and may only be provided with restrictions (e.g., anonymized data).

Acknowledgments: The authors wish to acknowledge Sunghyun Yoon of Jungja Running Club, Jung-Ae Oh of Jungja 2 Dong Gateball Club, and all the participants for their help in data collection.

Conflicts of Interest: The authors declare no conflict of interest. The funders had no role in the design of the study; in the collection, analyses, or interpretation of data; in the writing of the manuscript, or in the decision to publish the results. 


\section{References}

1. Bell, S.L.; Audrey, S.; Gunnell, D.; Cooper, A.; Campbell, R. The Relationship between Physical Activity, Mental Wellbeing and Symptoms of Mental Health Disorder in Adolescents: A Cohort Study. Int. J. Behav. Nutr. Phys. Act. 2019, 16, 138. [CrossRef] [PubMed]

2. Nielsen, J.B. How We Walk: Central Control of Muscle Activity during Human Walking. Neuroscientist 2003, 9, 195-204. [CrossRef]

3. Nilsson, J.; Thorstensson, A.; Halbertsma, J.N. Changes in Leg Movements and Muscle Activity with Speed of Locomotion and Mode of Progression in Humans. Acta Physiol. Scand. 1985, 123, 457-475. [CrossRef] [PubMed]

4. Michael, M. These Boots Are Made for Walking...: Mundane Technology, the Body and Human-Environment Relations. Body Soc. 2000, 6, 107-126. [CrossRef]

5. Zhang, K.; de Silva, C.W.; Fu, C. Sensor Fusion for Predictive Control of Human-Prosthesis-Environment Dynamics in Assistive Walking: A Survey. arXiv 2019, arXiv:1903.07674.

6. Matos Wunderlich, F. Walking and Rhythmicity: Sensing Urban Space. J. Urban Des. 2008, 13, 125-139. [CrossRef]

7. Gauvin, L.; Richard, L.; Kestens, Y.; Shatenstein, B.; Daniel, M.; Moore, S.D.; Mercille, G.; Payette, H. Living in a Well-Serviced Urban Area Is Associated with Maintenance of Frequent Walking among Seniors in the VoisiNuAge Study. J. Gerontol. Ser. B Psychol. Sci. Soc. Sci. 2012, 67, 76-88. [CrossRef]

8. Jeong, D.Y.; Han, S.H.; Kwahk, J.; Park, J.; Lee, M.; Park, K.; Kim, J.H.; Jang, H.; Jeong, D. B3-2 Suggesting Pedestrian Experience Principles of the Mobility Handicapped. Jpn. J. Ergon. 2017, 53, S412-S415. [CrossRef]

9. Forsyth, A. What Is a Walkable Place? The Walkability Debate in Urban Design. Urban Des. Int. 2015, 20, 274-292. [CrossRef]

10. Ewing, R.; Handy, S. Measuring the Unmeasurable: Urban Design Qualities Related to Walkability. J. Urban Des. 2009, 14, 65-84. [CrossRef]

11. Lee, G.; Choi, B.; Jebelli, H.; Ahn, C.R.; Lee, S. Wearable Biosensor and Collective Sensing-Based Approach for Detecting Older Adults' Environmental Barriers. J. Comput. Civ. Eng. 2020, 34, 04020002. [CrossRef]

12. Kim, H.; Ahn, C.R.; Yang, K. A People-Centric Sensing Approach to Detecting Sidewalk Defects. Adv. Eng. Inform. 2016, 30, 660-671. [CrossRef]

13. Dawson, J.; Hillsdon, M.; Boller, I.; Foster, C. Perceived Barriers to Walking in the Neighborhood Environment: A Survey of Middle-Aged and Older Adults. J. Aging Phys. Act. 2007, 15, 318-335. [CrossRef]

14. Lockett, D.; Willis, A.; Edwards, N. Through Seniors' Eyes: An Exploratory Qualitative Study to Identify Environmental Barriers to and Facilitators of Walking. Can. J. Nurs. Res. Arch. 2005, 37, 48-65.

15. Salmon, J.; Salmon, L.; Crawford, D.A.; Hume, C.; Timperio, A. Associations among Individual, Social, and Environmental Barriers and Children's Walking or Cycling to School. Am. J. Health Promot. 2007, 22, 107-113. [CrossRef]

16. Li, C.; Chi, G.; Jackson, R. Perceptions and Barriers to Walking in the Rural South of the United States: The Influence of Neighborhood Built Environment on Pedestrian Behaviors. Urban Des. Int. 2015, 20, 255-273. [CrossRef]

17. Yoon, J.; Chun, J.; Kim, H. Investigating the Relation between Walkability and the Changes in Pedestrian Policy through Wearable Sensing. Sustainability 2020, 12, 10447. [CrossRef]

18. Kim, B.; Yoo, M.; Park, K.C.; Lee, K.R.; Kim, J.H. A Value of Civic Voices for Smart City: A Big Data Analysis of Civic Queries Posed by Seoul Citizens. Cities 2021, 108, 102941. [CrossRef]

19. Bereitschaft, B. Walk Score®versus Residents' Perceptions of Walkability in Omaha, NE. J. Urban. Int. Res. Placemaking Urban Sustain. 2018, 11, 412-435. [CrossRef]

20. Deehr, R.C.; Shumann, A. Active Seattle: Achieving Walkability in Diverse Neighborhoods. Am. J. Prev. Med. 2009, 37, S403-S411 [CrossRef]

21. Battista, G.A.; Manaugh, K. Using Embodied Videos of Walking Interviews in Walkability Assessment. Transp. Res. Rec. 2017, 2661, 12-18. [CrossRef]

22. Resch, B.; Puetz, I.; Bluemke, M.; Kyriakou, K.; Miksch, J. An Interdisciplinary Mixed-Methods Approach to Analyzing Urban Spaces: The Case of Urban Walkability and Bikeability. Int. J. Environ. Res. Public Health 2020, 17, 6994. [CrossRef]

23. Lee, G.; Choi, B.; Ahn, C.R.; Lee, S. Wearable Biosensor and Hotspot Analysis-Based Framework to Detect Stress Hotspots for Advancing Elderly's Mobility. J. Manag. Eng. 2020, 36, 04020010. [CrossRef]

24. Feldstein, L.M. Policy and Legislation for Healthy Places. In Making Healthy Places; Springer: Washington, DC, USA, 2011; pp. 271-286.

25. Heinrich, K.M.; Stephen, M.O.; Vaughan, K.B.; Kellogg, M. Kansas Legislators Prioritize Obesity but Overlook Nutrition and Physical Activity Issues. J. Public Health Manag. Pract. 2013, 19, 139-145. [CrossRef] [PubMed]

26. Campbell, A.T.; Eisenman, S.B.; Lane, N.D.; Miluzzo, E.; Peterson, R.A.; Lu, H.; Zheng, X.; Musolesi, M.; Fodor, K.; Ahn, G.-S. The Rise of People-Centric Sensing. IEEE Internet Comput. 2008, 12, 12-21. [CrossRef]

27. Campbell, A.T.; Eisenman, S.B.; Lane, N.D.; Miluzzo, E.; Peterson, R.A. People-Centric Urban Sensing. In Proceedings of the 2nd Annual International Workshop on Wireless Internet, Boston, MA, USA, 2-5 August 2006; Association for Computing Machinery: New York, NY, USA, 2006; p. 18-es.

28. Hussain, A.; Wenbi, R.; da Silva, A.L.; Nadher, M.; Mudhish, M. Health and Emergency-Care Platform for the Elderly and Disabled People in the Smart City. J. Syst. Softw. 2015, 110, 253-263. [CrossRef]

29. Lu, H.; Pan, W.; Lane, N.D.; Choudhury, T.; Campbell, A.T. SoundSense: Scalable Sound Sensing for People-Centric Applications on Mobile Phones. In Proceedings of the 7th International Conference on Mobile Systems, Applications, and Services, Kraków, Poland, 22-25 June 2009; Association for Computing Machinery: New York, NY, USA, 2009; pp. 165-178. 
30. Sofia, R.; Firdose, S.; Lopes, L.A.; Moreira, W.; Mendes, P. NSense: A People-Centric, Non-Intrusive Opportunistic Sensing Tool for Contextualizing Nearness. In Proceedings of the 2016 IEEE 18th International Conference on e-Health Networking, Applications and Services (Healthcom), Munich, Germany, 14-17 September 2016; IEEE: Piscataway, NJ, USA, 2016 ; pp. 1-6.

31. Higashino, T.; Uchiyama, A. A Study for Human Centric Cyber Physical System Based Sensing-toward Safe and Secure Urban Life-. In Proceedings of the International Workshop on Information Search, Integration, and Personalization, Sapporo, Japan, 11-13 October 2012; Springer: Berlin, Heidelberg, 2012; pp. 61-70.

32. Bayındır, L. A Survey of People-Centric Sensing Studies Utilizing Mobile Phone Sensors. J. Ambient Intell. Smart Environ. 2017, 9 , 421-448. [CrossRef]

33. Jansi, K.R.; Sandhia, G.K.; KasmirRaja, S.V. A Reliable Cloud Based Framework for Large Scale Urban Sensing Systems. In Smart Intelligent Computing and Communication Technology; IOS Press: Amsterdam, The Netherlands, 2021; pp. 67-72.

34. Zeile, P. Defining and Assessing Walkability: A Concept for an Integrated Approach Using Surveys, Biosensors and Geospatial Analysis. Urban Dev. Issues 2019, 62, 5-15.

35. Ahn, C.; Ham, Y.; Kim, J.; Kim, J. A Digital Twin City Model for Age-Friendly Communities: Capturing Environmental Distress from Multimodal Sensory Data. In Proceedings of the 53rd Hawaii International Conference on System Sciences, Maui, HI, USA, 7-10 January 2020.

36. Kim, J.; Yadav, M.; Chaspari, T.; Ahn, C.R. Saliency Detection Analysis of Collective Physiological Responses of Pedestrians to Evaluate Neighborhood Built Environments. Adv. Eng. Inform. 2020, 43, 101035. [CrossRef]

37. Zanwar, P.; Kim, J.; Kim, J.; Manser, M.; Ham, Y.; Chaspari, T.; Ahn, C.R. Use of Connected Technologies to Assess Barriers and Stressors for Age and Disability-Friendly Communities. Front. Public Health 2021, 9, 578832. [CrossRef]

38. Kim, H.; Ahn, C.R.; Yang, K. Identifying Safety Hazards Using Collective Bodily Responses of Workers. J. Constr. Eng. Manag. 2017, 143, 04016090. [CrossRef]

39. Kim, H. Wearable Sensor Data-Driven Walkability Assessment for Elderly People. Sustainability 2020, 12, 4041. [CrossRef]

40. Leão, A.L.F.; Abonizio, H.Q.; Reis, R.S.; Kanashiro, M. Walkability Variables: An Empirical Study in Rolândia-PR, Brazil. Ambiente Construído 2020, 20, 475-488. [CrossRef]

41. Tan, Y.Z.; Wu, C.F. The Laws of the Information Entropy Values of Land Use Composition. J. Nat. Resour. 2003, 18, $112-117$.

42. Zhao, J.; XU, J.; MEI, A.; WU, J.; ZHOU, J. A Study on the Information Entropy and Fractal Dimension of Land Use Structure and Form in Shanghai. Geogr. Res. 2004, 2, 137-146.

43. Li, C.; Zhang, F.; Zhu, T.; Feng, T.; An, P. Evaluation and Correlation Analysis of Land Use Performance Based on Entropy-Weight TOPSIS Method. Trans. Chin. Soc. Agric. Eng. 2013, 29, 217-227.

44. Zhang, X.; Yu, Q.; Wang, Y. Fuzzy Evaluation of Crowd Safety Based on Pedestrians' Number and Distribution Entropy. Entropy 2020, 22, 832. [CrossRef]

45. Procházka, J.; Olševičová, K. Monitoring Lane Formation of Pedestrians: Emergence and Entropy. In Proceedings of the Asian Conference on Intelligent Information and Database Systems, Bali, Indonesia, 23-25 March 2015; Springer: Cham, Switzerland, 2015; pp. 221-228.

46. Rangel-Huerta, A.; Ballinas-Hernández, A.L.; Muñoz-Meléndez, A. An Entropy Model to Measure Heterogeneity of Pedestrian Crowds Using Self-Propelled Agents. Phys. A Stat. Mech. Its Appl. 2017, 473, 213-224. [CrossRef]

47. Tageldin, A.; Zaki, M.H.; Sayed, T. Examining Pedestrian Evasive Actions as a Potential Indicator for Traffic Conflicts. IET Intell. Transp. Syst. 2017, 11, 282-289. [CrossRef]

48. Qiao, Z.; Zhao, L.; Gu, L.; Jiang, X.; Li, R.; Ge, L. Research on Abnormal Pedestrian Trajectory Detection of Dynamic Crowds in Public Scenarios. IEEE Sens. J. 2021, 21, 23046-23054. [CrossRef]

49. O'Connor, C.M.; Thorpe, S.K.; O’Malley, M.J.; Vaughan, C.L. Automatic Detection of Gait Events Using Kinematic Data. Gait Posture 2007, 25, 469-474. [CrossRef]

50. Zmeskal, O.; Dzik, P.; Vesely, M. Entropy of Fractal Systems. Comput. Math. Appl. 2013, 66, 135-146. [CrossRef]

51. Hirsh, J.B.; Mar, R.A.; Peterson, J.B. Psychological Entropy: A Framework for Understanding Uncertainty-Related Anxiety. Psychol. Rev. 2012, 119, 304. [CrossRef]

52. Shannon, C.E. A Mathematical Theory of Communication. Bell Syst. Tech. J. 1948, 27, 623-656. [CrossRef]

53. Bobko, P. Correlation and Regression: Applications for Industrial Organizational Psychology and Management; Sage Publications: Newbury Park, CA, USA, 2001.

54. Holley, J.W.; Berhagen, K.-E. A Note on the Use of the Point Biserial Correlation Coefficient; Lund University: Lund, Sweden, 1969.

55. Yang, K.; Ahn, C.R. Inferring Workplace Safety Hazards from the Spatial Patterns of Workers' Wearable Data. Adv. Eng. Inform. 2019, 41, 100924. [CrossRef]

56. Lee, B.; Hwang, S.; Kim, H. The Feasibility of Information-Entropy-Based Behavioral Analysis for Detecting Environmental Barriers. Int. J. Environ. Res. Public Health 2021, 18, 11727. [CrossRef] [PubMed]

57. Lane, N.D.; Eisenman, S.B.; Musolesi, M.; Miluzzo, E.; Campbell, A.T. Urban Sensing Systems: Opportunistic or Participatory? In Proceedings of the 9th Workshop on Mobile Computing Systems and Applications, Napa Valley, CA, USA, 25-26 February 2008; ACM: Singapore, 2008; pp. 11-16. 
58. Moreira, W.; Mendes, P. Pervasive Data Sharing as an Enabler for Mobile Citizen Sensing Systems. Commun. Mag. IEEE 2015, 53, 164-170. [CrossRef]

59. Iwarsson, S.; Nygren, C.; Oswald, F.; Wahl, H.-W.; Tomsone, S. Environmental Barriers and Housing Accessibility Problems over a One-Year Period in Later Life in Three European Countries. J. Hous. Elder. 2006, 20, 23-43. [CrossRef] 